\title{
A NOTE ON GENTRE-BY-FINITE-EXPONENT VARIETIES OF GROUPS
}

\author{
NARAIN GUPTA and AKBAR RHEMTULLA \\ (Received 4 March 1969, revised 1 May 1969) \\ To Bernhard Hermann Neumann on his 60th birthday \\ Communicated by G. E. Wall
}

We refer the reader to Hanna Neumann [7] for notation and other undefined terms. Let $\mathfrak{A}(n), \mathfrak{B}(n)$ and $\mathfrak{C}(n)$ denote the varieties of groups defined by the laws $(x y)^{n}=x^{n} y^{n},[x, y]^{n}=1$ and $\left[x, y^{n}\right]=1$ respectively, where $n$ is an integer. $\mathfrak{U}(n)$-groups were termed " $n$-abelian" by R. Baer [1] and have been a subject matter of investigation by various authors (see [3], [5], [6] and the references therein). Recently Kalužnin [5] has shown that $\mathfrak{A}(n)=\mathfrak{A} \vee \mathfrak{B}_{n} \vee \mathfrak{B}_{n-1}(n \neq 0,1)$, thus clarifying the relationship between $\mathfrak{A}(n)$ and the familiar varieties. From the elementary inequalities

(1) $\mathfrak{A}(n)=\mathfrak{A}(1-n) \leqq\left[\mathfrak{B}_{n(n-1)}, \mathbb{E}\right]=\mathbb{E}(n(n-1)) \leqq \mathfrak{A P}_{n(n-1)} \quad(n \neq 0,1)$

it is easily deduced that

$$
\mathfrak{A}(n) \leqq \mathfrak{B}(n(n-1))
$$

(see for instance [5]). If $G=C_{m} W r C_{\infty}$, then clearly $G \in \mathfrak{B}(m)$ but $G \notin \mathbb{C}\left(m^{*}\right)$ for any $m^{*} \neq 0$ and hence $G \notin \mathfrak{A}\left(m^{*}\right)$ for any $m^{*} \neq 0,1$. Thus $\mathfrak{B}(m) \$ \mathfrak{C}\left(m^{*}\right)$ and $\mathfrak{B}(m) \$ \mathfrak{A}\left(m^{*}\right)$. It is also easy to see that in general $\mathfrak{S}(n(n-1)) \$ \mathfrak{A}(n)$ (see for instance [6] §5.1) and we are led to ask

QUESTION 1: Does there exist for each positive integer $m$, an integer $f(m)$ such that $\mathbb{E}(m) \leqq \mathfrak{A}(f(m))$ ?

If $m$ is such that $B_{2, m}$ (the unrestricted Burnside group of exponent $m$ on 2 generators) is finite, then for a group $G=\langle x, y\rangle$ in $\mathbb{E}(m)$ one has $G / Z(G)$ finite and by a well-known theorem of Schur [8] (page 26) $G^{\prime}$ is finite, say, of exponent $m^{*}$. Now for a suitable $u$ in $G^{\prime}$ we have that $(x y)^{m m^{*}}=\left(x^{m} y^{m} u\right)^{m^{*}}=x^{m m^{*}} y^{m m^{*}}$; hence $\mathbb{C}(m) \leqq \mathfrak{A}\left(m m^{*}\right)$. In particular Question 1 has affirmative answers for $m=2,3,4$ and 6 . However not relying on the solution of the Burnside problem we are able to prove

THEOREM 1. (i) $\mathfrak{C}(2) \leqq \mathfrak{A}(4)$, (ii) $\mathfrak{C}(3) \leqq \mathfrak{A}(9)$, (iii) $\cdot \mathfrak{E}(4) \leqq \mathfrak{A}(32)$. 
Proof: We note that the laws $\left[x, y^{n}\right]=1$ and $(x y)^{n}=(y x)^{n}$ are equivalent.

(i) The law $\left[x, y^{2}\right]=1$ implies $[x, y, z]=1$ and so also $[x, y]^{2}=1$. Thus $(x y)^{4}=\left((x y)^{2}[x, y]\right)^{2}=\left((y x)^{2}[x, y]\right)^{2}=\left(y x^{2} y\right)^{2}=\left(y^{2} x^{2}\right)^{2}=x^{4} y^{4}$.

(ii) $(x y)^{9}=\left((x y)^{3}[x, y]\right)^{3}[y, x]^{3}=\left((y x)^{3}[x, y]\right)^{3}(y, x]^{3}=$ $((y x y)(x x y))^{3}[y, x]^{3}=((x x y)(y x y))^{3}[y, x]^{3}=\left(x^{3} y^{3}[x, y]\right)^{3}[y, x]^{3}=x^{9} y^{9}$.

(iii) $\left(x y^{2}\right)^{16}=\left(\left(x y^{2}\right)^{4}\left[x, y^{2}\right]\right)^{4}\left[y^{2}, x\right]^{4}=\left(\left(y^{2} x\right)^{4}\left[x, y^{2}\right]\right)^{4}\left[y^{2}, x\right]^{4}=$ $\left(\left(y^{2} x\right)^{3} x y^{2}\right)^{4}\left[y^{2}, x\right]^{4}=\left(x^{2} y^{4}\left(x y^{2}\right)^{2}\right)^{4}\left[y^{2}, x\right]^{4}=\left(x^{4} y^{8}\left[x, y^{2}\right]\right)^{4}\left[y^{2}, x\right]^{4}=x^{16} y^{32}$. Replace $x$ by $x^{-1}$ and $y$ by $x y$ to get $(y x y)^{16}=x^{-16}(x y)^{32}$. Thus

$$
(x y)^{32}=x^{16}(y(x y))^{16}=x^{16}\left(x y^{2}\right)^{16}=x^{32} y^{32} \text {. }
$$

It follows from (2) that a torsion-free $\mathfrak{U}(n)$-group is abelian (since a torsion-free $\mathfrak{B}(n)$-group is abelian). Here we ask

QUESTION 2: Is every torsion-free $\mathfrak{E}(n)$-group abelian?

This question is not new and in fact there is an outstanding conjecture that this question has an affirmative answer. Obviously Question 2 has positive answer for those integers for which Question 1 has positive answer. Further, since by Schur's Theorem a torsion-free centre-by-finite group is abelian, it follows that a torsion-free locally soluble $\mathbb{E}(n)$-group is abelian. Without any such assumption we are able to prove

THEOREM 2. A torsion-free $\mathbb{C}(n)$-group is abelian for $n=2^{k} 3^{l}$ $(k \geqq 0, l=0,1)$.

Proof: Let $G$ be a torsion-free group in $5\left(2^{k} 3^{l}\right)$. We prove by reverse induction on $j \in\{k, \cdots, 0\}$ that $G \in \mathbb{C}\left(2^{j} 3^{l}\right)$. For $j=k$ the result is given. Assume $G \in \mathbb{C}\left(2^{i+1} 3^{i}\right) \quad(0 \leqq i<k)$. We show that $\left[x, y^{2^{i} 3^{l}}\right]=1$ for all $x, y \in G$. Put $z=y^{2^{i} 3^{l}}$, so that by induction hypothesis, $\left[x, z^{2}\right]=1$. Thus $[x, z]^{-1}=[x, z]^{z}$. But this implies that $[x, z]^{2^{i+1} 3^{i}}=[x, z]^{2^{1+1} 3^{l_{3}}}=[x, z]^{-2^{i+1} 3^{l}}$. Hence $[x, z]^{2^{i+2} 3^{i}}=1$. Since $G$ is torsion-free, $[x, z]=1$ and $G \in \mathbb{C}\left(2^{i} 3^{l}\right)$. Thus $G \in \mathbb{E}\left(2^{j} 3^{l}\right)$ for all $j \in\{k, \cdots, 0\}$, and $G \in \mathbb{E}(1)=\mathfrak{A}$ or $\mathbb{S}(3)$ depending on whether $l=0$ or $l=1$. In both cases $G$ is abelian by Theorem 1 .

REMARK 1. If $G \in \mathfrak{A}(n)$, then for any $x, y \in G$,

$$
\left(x^{-1} y^{-1} x y\right)^{n}=\left(x^{-1} y^{-1}\right)^{n}(x y)^{n}=(y x)^{-n}(x y)^{n} \text {. }
$$

Thus by Kalužnin's Theorem 3 we have

$$
\mathfrak{U}(n) \wedge \mathfrak{B}(n)=\mathfrak{A}(n) \wedge \mathfrak{C}(n)=\mathfrak{U} \vee \mathfrak{B}_{n} .
$$

REMARK 2. It seems worthwhile to remark that if $G$ is a torsion-free Engel group in $\mathbb{C}(n),(n \neq 0)$ then for any two elements $x, y$ in $G$, either $[x, y]=1$ or there exists an integer $r \geqq 1$ such that $[x, r y] \neq 1$ but 
$[x,(r+1) y]=1$. In the latter case, $1=\left[x,(r-1) y, y^{n}\right]=[x, r y]^{n}=[x, r y]$ gives a contradiction. Thus in $\mathbb{S}(n)$ torsion-free Engel groups are abelian.

Remark 3. In [3] Durbin considered the problem of characterizing those sequences $\left\{n_{1}, \cdots, n_{t}\right\}$ of integers for which it is true that $\bigwedge_{k=1}^{t} \mathfrak{A}\left(n_{k}\right)=\mathfrak{A}$. If $\mathfrak{B}$ denotes the class of all groups of finite exponent, then he proves that $\mathfrak{B} \wedge\left(\bigwedge_{k=1}^{t} \mathfrak{A}\left(n_{k}\right)\right)<\mathfrak{A}$ if and only if $\left(\left(\begin{array}{c}n_{1} \\ 2\end{array}\right), \cdots,\left(\begin{array}{c}n_{t} \\ 2\end{array}\right)\right)=1$, where $\left(\begin{array}{c}n_{k} \\ 2\end{array}\right)=$ $\frac{1}{2} n_{k}\left(n_{k}+1\right)$. He shows further that the hypothesis of finite exponent can be replaced by "periodicity" in the special case $\{n, n+2\}$. We complete the discussion on Durbin's problem by proving,

THEOREM 3. $\bigwedge_{k=1}^{t} \mathfrak{A}\left(n_{k}\right)=\mathfrak{A}$ if and only if $\left(\left(\begin{array}{c}n_{1} \\ 2\end{array}\right), \cdots,\left(\begin{array}{c}n_{t} \\ 2\end{array}\right)\right)=1$.

Proof: The "only if" part of the theorem follows from Durbin's proof. For the rest of the proof we first notice from (1), that

$$
\begin{aligned}
\bigwedge_{k=1}^{t} \mathfrak{A}\left(n_{k}\right) & \leqq \bigwedge_{k=1}^{t}\left[\mathfrak{B}_{n_{k}\left(n_{k}-1\right)}, \mathbb{E}\right] \\
& \leqq\left[\bigwedge_{k=1}^{t} \mathfrak{B}_{n_{k}\left(n_{k}-1\right)}, \mathbb{E}\right]=\left[\mathfrak{B}_{2}, \mathbb{E}\right] \leqq \mathfrak{N}_{2} .
\end{aligned}
$$

But groups in $\mathfrak{R}_{2}$ satisfy the law $(x y)^{n}=x^{n} y^{n}[y, x]^{\left(\begin{array}{c}n \\ 2\end{array}\right)}$ for every integer $n$. Thus $\bigwedge_{k=1}^{t} \mathfrak{A}\left(n_{k}\right)=\mathfrak{A}$, as was required.

REMARK 4. In our initial proof of Theorem 3 we made use of the following lemma which seems to be of independent interest (c.f. [7] page 39 and [2].

LEMMA. $\mathfrak{N}_{c}\left(\bigwedge_{k=1}^{t} \mathfrak{B}_{m_{k}}\right)=\bigwedge_{k=1}^{t} \mathfrak{R}_{c} \mathfrak{B}_{m_{k}}$

Proof: For positive integers $c, m, n$ let $G \in \mathfrak{R}_{c} \mathfrak{B}_{m} \wedge \mathfrak{R}_{c} \mathfrak{B}_{n}$. Then $\left[x_{1}^{m}, \cdots, x_{c+1}^{m}\right]=\left[x_{1}^{n}, \cdots, x_{c+1}^{n}\right]=1$ for all $x_{i} \in G$. If $d=(m, n)$, both $\left[x_{1}^{d}, \cdots, x_{c+1}^{d}\right)^{(m / d)^{c+1}}$ and $\left[x_{1}^{d}, \cdots, x_{c+1}^{d}\right]^{(n / d)^{c+1}}$ lie in $\left(G^{d}\right)_{(c+2)}$, where $G^{d}=\left\langle x^{d} ; x \in G\right\rangle$. Thus $\left(G^{d}\right)_{(c+1)}=\left(G^{d}\right)_{(c+2)}$. On the other hand $G^{d} \equiv G^{m} G^{n}$ is nilpotent since $G^{m}, G^{n} \in \Re_{c}$. Thus $\left(G^{d}\right)_{(c+1)}=1$ and $G \in \mathfrak{R}_{c} \mathfrak{B}_{d}$. This proves $\mathfrak{R}_{c}\left(\mathfrak{B}_{n} \wedge \mathfrak{B}_{n}\right)=\mathfrak{R}_{c} \mathfrak{B}_{m} \wedge \mathfrak{R}_{c} \mathfrak{B}_{n}$ and the lemma follows.

REviark 5. In the concluding section of his paper [3], Durbin raised the following number theoretic question: Does there exist, for each positive integer $t$, a set $\left\{n_{1}, \cdots, n_{t}\right\}$ of integers satisfying $\left(\left(\begin{array}{c}n_{1} \\ 2\end{array}\right), \cdots,\left(\begin{array}{c}n_{t} \\ 2\end{array}\right)\right)=1$ such that no proper subset satisfies this property? We give an affirmative answer to this question by giving a process of constructing such integers. This construction is due to T. J. Dickson whose co-operation is gratefully acknowledged. 
For $t=2$, the set $\{2,3\}$ will do. For $t>2$ we first choose a set $p_{1}$, $p_{2}, \cdots, p_{t}$ of primes as follows: choose $p_{1}=2, p_{2}=3$ and for $3 \leqq i \leqq t$, choose $p_{i}$ to be of the form $l_{i} p_{1} p_{2} \cdots p_{i-1}+1$ for some integer $l_{i} \geqq 3$. This is possible by Dirichlet's Theorem (see for instance [4] page 13). Thus $p_{i} \equiv 1\left(p_{j}\right)$ for $j=1, \cdots, i-1$. Let $p_{i}^{\prime}=\prod_{j \neq i} p_{j}$ and define $n_{i}=2 p_{i}^{\prime}+1$. It is now routine to show that the set $\left\{n_{1}, \cdots, n_{t}\right\}$ has the required properties.

\section{Acknowledgement}

We thank the referee for drawing our attention to Kalužnin's paper and for his many useful suggestions.

\section{References}

[1] R. Baer, 'Factorization of $n$-soluble and n-nilpotent groups', Proc. Amer. Math. Soc. 4 (1953), $15-26$.

[2] V. Dlab, 'A note on powers of a group', Acta. Sci. Math. (Szeged) 25 (1964), 177-178.

[3] J. R. Durbin, 'Commutativity and $n$-abelian groups', Math. Zeitschr. 98 (1967), 89-92.

[4] G. H. Hardy and E. M. Wright, Theory of Numbers, (Oxford 1965).

[5] L. A. Kalužnin, 'The structure of $n$-abelian groups' (Russian), Mat. Zametki 2 (1967), $455-462$.

[6] G. A. Karasev, 'The concept of n-nilpotent groups', Sibirskii Mat. Zhurnal 7 (1966), 1014-1032. English Translation pp. 808-821.

[7] Hanna Neumann, Varieties of Groups, (Springer-Verlag 1967).

[8] I. Schur, 'Uber die Darstellung der endlichen Gruppen durch gebrochene Jineare substitutionen', $J$, reine angew. Math. 27 (1904), 20-50.

University of Manitoba

and

University of Alberta, Edmonton 